Annals of Plant and Soil Research 23(1): 116-122 (2021)

https://doi.org/10.47815/apsr.2021.10041

\title{
Combining ability and gene action for seed yield and its component traits in green gram (Vigna radiata L.)
}

\author{
PUSHPENDRA SINGH ${ }^{\star}$, SURENDRA SINGH, C.B.S. JATAV ${ }^{1}$, M.K. BHARGAVA ${ }^{2}$, AND PUNEET \\ KUMAR $^{3}$
}

Department of Genetics \& Plant Breeding, Brahmanand Mahavidyalaya, Rath, (Hamirpur,(UP.)-210431

Received: December, 2020; Revised accepted: January, 2021

\begin{abstract}
An investigation to study of combining ability and gene action was conducted on green gram for seed yield per plant and its component traits. For this study, line $x$ tester design of mating was used taking six genotypes as lines and four genotypes as testers. The variance analysis revealed significant differences for different sources of variation for most of the traits. The higher specific combining ability (SCA) values were compared to general combining ability (GCA) values. The higher degree of dominance indicated preponderance of non additive gene action. The dominance variance was predominant for all the characters except for pods per plant indicating the major role of non-additive gene action and it was highest for harvest index (HI) followed by plant height and days to 50\% flowering. The parents, viz., BM 4 and JM 721 among line and AKM 8802 among testers exhibited positive significant effects and higher per se performance for seed yield per plant and other component traits and hence considered as superior parents. The hybrids viz., BM4 x PDM1, JM $10 \times A K M$ 8802, JM 721 x PDM1, ML131 x HUM 1 and TARM 2 x TJM 3 were observed as superior specific combiners for seed yield per plant and its component traits. These hybrids or cross combinations might be utilized for further improvement of seed yield in green gram.
\end{abstract}

Keywords: Green gram, line x tester, combining ability, GCA, SCA, degree of dominance

\section{INTRODUCTION}

In a country like India, pulses, also called as grain legumes are the cheapest and most widely consumed source of protein. They are also used as feed and fodder for cattle and other pet animals. They contain a very good amount of protein ranging from 20 to $30 \%$, which is 2.0 to 3.0 times greater than that of cereals. Pulses are also a good source of lysine which is an essential amino acid for the human body. Among legumes, green gram (Vigna radiata (L.) is the third most cultivated annual crop of Asia due to high protein content, broad adaptation, lesser agronomical requirement and high ability to increase soil fertility. It belongs to the family Fabaceae with chromosome number $2 n=22$. In literature, both additive and nonadditive genetic systems controlling grain yield and yield-related traits in green gram have been reported by Barad et al. (2008), Sathya and Jayamani (2011), Sujatha and Kajidoni (2013) and Suresh (2014). However, the major part of genetic variation for yield and its components was conditioned due to a higher magnitude of nonadditive genetic effects (Sathya and Jayamani (2011); Suresh (2014) and Narsimhulu et al (2014). The yield of green gram is low and limited success achieved so far in augmenting its yield despite high demand. To enhance the present yield levels, a systematic varietal improvement through hybridization and exploitation of generated variability through recombination breeding must be followed. Combining ability is a powerful tool to select good combiners and thus select the appropriate parental lines for hybridization programmes. Besides, information on the nature of gene action will be helpful to develop an efficient crop improvement programme. Grain yield and several yield contributing characters lack stability due to strong environmental influence suggesting the need for breeding for a specific environment. The present investigation was, therefore, undertaken to assess the nature of gene action involved and combining the ability of parental genotypes for various traits for evolving genotypes for better in productivity. 


\section{MATERIAL AND METHODS}

Six lines were crossed with four testers in line $\times$ tester mating design at Brahmanand Mahavidyalaya Research Farm, Rath, Hamirpur (UP), during zaid season of 2018. All the genotypes (24 hybrids and 10 parents) were grown in a randomized block design (RBD) with three replications during zaid, 2019. Each genotype was grown in a single row of $3.5 \mathrm{~m}$ length with a spacing of $40 \mathrm{~cm}$ between rows and $10 \mathrm{~cm}$ plant to plant. The guard rows were provided on all sides of each block. All recommended agronomical and plant protection measures were followed to raise healthy crop. Data were recorded on five randomly selected plants from each net plot of parents and F1s in all the three replications. Mean value per plant basis were recorded for various characters viz., days to $50 \%$ flowering, days to maturity, plant height, primary branches / plant, pods / cluster, clusters / plant, pods per plant, seeds / pod, seed weight / pod, biological yield / plant, seed yield / plant, 100 seed weight and harvest index. The observations on days to flowering and days to maturity were recorded on plot basis. The mean data were analyzed to compute the combining ability effect and their variance. The significance of gca lines, gca testers and sca effects were tested by comparing with respective $C D$ values.

\section{RESULTS AND DISCUSSION}

The analysis of data (Table 1 to 4 .) revealed significant differences in parents for all characters indicating a considerable amount of variability among the parents for various characters used in this study. The crosses showed highly significant differences for all the characters except for seeds / pod, which indicate the sufficient variability among the crosses for most of the traits. Crosses vs. parent comparisons were significant for all the characters except for days to maturity, pods / cluster, seeds / pod, seed weight and 100-seed weight revealing good scope for the manifestation of Heterosis for most of the traits studied. The effect of crosses was partitioned into lines, testers and their interactions. Analysis of variance for combining ability (Table 1) indicated that mean squares due to lines and mean squares due to testers were significant for all the characters under study. The mean squares due to lines $\times$ testers were observed to be significant for all the 13 characters except pods / plant and seeds / pod indicating the involvement of specific combining ability in the expression of these traits. These trends indicated that the experimental material possessed considerable variability and that additive and nonadditive gene actions were involved in the genetic control of various characters. The importance of dominance variance for most of the traits was also reported by Bainade et al. (2014) and Narsimhulu et al. (2014). The significant mean square of lines and testers indicated the prevalence of additive variance for the yield and its component traits. The occurrence of both additive and non-additive gene effects for yield and important yield component traits in green gram were reported by Anbumalarmathi et al. (2005), Marappa (2008), Barad et al. (2008), Sathya and Jayamani (2011), Sujatha and Kajjidoni (2013), Suresh (2014), and Narsimhulu et al.(2014). Furthermore, the variance component due to lines was higher in magnitude than that of due to testers for pods per cluster, clusters per plant, pods per plot, biological yield per plant 100 -seed weight and seed yield per plant which indicate a greater contribution of lines towards the ó ${ }^{2} g c a$ for these traits.

The analysis revealed higher values of sca variance than the gca variance, which indicated preponderance of nonadditive gene action for all the characters under study. The ratio of variances due to gca and sca (ó ${ }^{2} \mathrm{gca} /$ ó $^{2}$ sca) ranged from -0.003 to 0.733 , confirming the major role of non-additive gene action for all the traits under study (Table 2). From the estimates of additive and dominance variance, it was observed that dominance variance was predominant for all the characters except for pods per plant indicating the major role of nonadditive gene action and was highest for harvest index $(\mathrm{HI})$ followed by plant height and days to $50 \%$ flowering. The present results indicated the preponderance of nonadditive gene action in the expression of most of the characters studied and scope for the exploitation of nonadditive genetic variation for traits through heterotic breeding followed by selection at later generations. These results were in line with the findings of Sujatha and Kajjidoni (2013), Suresh (2014), Narsimhulu et al. (2014), Bhavani et al (2016) and Swarna Latha et al. (2018). 
Table 1: ANOVA for combining ability in a Line $\times$ Tester analysis for yield and its component in green gram

\begin{tabular}{|c|c|c|c|c|c|c|c|c|c|c|c|c|c|c|}
\hline $\begin{array}{l}\text { Source of } \\
\text { variation }\end{array}$ & d.f. & $\begin{array}{c}\text { Days to } \\
50 \% \\
\text { flowering }\end{array}$ & $\begin{array}{l}\text { Days to } \\
\text { maturity }\end{array}$ & $\begin{array}{c}\text { Primary } \\
\text { branches } \\
\text { /plant }\end{array}$ & $\begin{array}{r}\text { Plant } \\
\text { height } \\
(\mathrm{cm})\end{array}$ & $\begin{array}{l}\text { Pods/ } \\
\text { cluster }\end{array}$ & $\begin{array}{l}\text { Clusters } \\
\text { per plant }\end{array}$ & $\begin{array}{l}\text { Pods } \\
\text { per } \\
\text { plant }\end{array}$ & $\begin{array}{l}\text { Biological } \\
\text { yield per } \\
\text { plant }\end{array}$ & $\begin{array}{l}\text { Seeds } \\
\text { per pod }\end{array}$ & $\begin{array}{l}\text { Seed } \\
\text { weight } \\
\text { per pod } \\
(\mathrm{g})\end{array}$ & $\begin{array}{c}100- \\
\text { seed } \\
\text { weight } \\
\text { (g) }\end{array}$ & $\begin{array}{c}\text { Harvest } \\
\text { Index } \\
(\%)\end{array}$ & $\begin{array}{c}\text { Seed yield } \\
\text { per } \\
\text { plant }(g)\end{array}$ \\
\hline Replication & 2 & 2.34 & 3.79 & 0.14 & 2.38 & 0.31 & $0.46^{* *}$ & 16.72 & $3.33^{*}$ & 1.10 & 0.0013 & 0.1017 & 1.33 & $0.88^{*}$ \\
\hline Parents & 9 & $72.08^{* *}$ & $64.68^{* *}$ & $0.48^{* *}$ & $32.68^{* *}$ & $2.42^{* *}$ & $2.07^{* *}$ & $76.76^{\star *}$ & $16.39^{* *}$ & $1.71^{* *}$ & $0.010^{* *}$ & $2.169^{\star * *}$ & $28.81^{* *}$ & $1.14^{* *}$ \\
\hline Crosses & 23 & $45.73^{\star *}$ & $41.47^{\star *}$ & $0.45^{* *}$ & $26.47^{\star *}$ & $2.30^{* *}$ & $2.16^{* *}$ & $\begin{array}{c}157.93^{*} \\
*\end{array}$ & $24.54^{* *}$ & $0.69^{*}$ & $0.0053^{\star *}$ & $0.5325^{\star *}$ & $49.53^{\star *}$ & $6.61^{* *}$ \\
\hline Line effect & 5 & $36.81^{* *}$ & $42.40^{\star *}$ & $0.73^{* *}$ & $23.38^{* *}$ & $6.48^{* *}$ & $7.21^{* *}$ & $\begin{array}{c}515.15^{*} \\
*\end{array}$ & $60.31^{\star *}$ & $0.93^{*}$ & $0.0209^{* *}$ & $1.1223^{\star *}$ & $34.50^{* *}$ & $13.64^{* *}$ \\
\hline Tester effect & 3 & $212.12^{* *}$ & $181.88^{* *}$ & $1.00^{* *}$ & $55.40^{\star *}$ & $3.78^{* *}$ & $1.85^{\star *}$ & $\begin{array}{c}259.35^{*} \\
*\end{array}$ & $21.71^{* *}$ & $1.63^{*}$ & $0.0022^{*}$ & $0.3224^{* *}$ & $65.35^{\star *}$ & $8.11^{* *}$ \\
\hline$L \times T$ effect & 15 & $15.43^{* *}$ & $13.08^{\star *}$ & $0.24^{* *}$ & $21.71^{* *}$ & $0.62^{* *}$ & $0.53^{*}$ & 18.58 & $13.18^{* *}$ & 0.43 & $0.0006^{*}$ & $0.3779^{\star *}$ & $51.38^{* *}$ & $3.97^{* *}$ \\
\hline $\begin{array}{c}\text { Cross. vs } \\
\text { Par. }\end{array}$ & 1 & 14.61 & $391.55^{\star}$ & $8.98^{*}$ & $318.57^{*}$ & 6.74 & $36.82^{*}$ & $\begin{array}{c}1253.74 \\
*\end{array}$ & $550.98^{* *}$ & 8.59 & 0.004 & 0.937 & $567.58^{*}$ & $176.35^{\star \star}$ \\
\hline Error & 46 & 0.28 & 0.26 & 0.01 & 0.67 & 0.06 & 0.07 & 4.20 & 0.09 & 0.10 & 0.0001 & 0.0077 & 1.80 & 0.02 \\
\hline Total & 71 & & & & & & & & & & & & & \\
\hline
\end{tabular}

* Significant at $5 \%$ level, ${ }^{* *}$ Significant at $1 \%$ level

Table 2: Estimation of GCA \& SCA, Additive and Dominance variances for yield and its attributing characters in green gram

\begin{tabular}{|c|c|c|c|c|c|c|c|c|c|c|c|c|c|}
\hline Type of variance & $\begin{array}{c}\text { Days to } \\
50 \% \\
\text { flowering }\end{array}$ & $\begin{array}{l}\text { Days to } \\
\text { maturity }\end{array}$ & $\begin{array}{c}\text { Primary } \\
\text { branches } \\
\text { /plant }\end{array}$ & $\begin{array}{l}\text { Plant } \\
\text { height } \\
(\mathrm{cm})\end{array}$ & $\begin{array}{l}\text { Pods/ } \\
\text { cluster }\end{array}$ & $\begin{array}{c}\text { Clusters } \\
\text { per } \\
\text { plant }\end{array}$ & $\begin{array}{c}\text { Pods } \\
\text { per } \\
\text { plant }\end{array}$ & $\begin{array}{c}\text { Biological } \\
\text { yield per } \\
\text { plant }\end{array}$ & $\begin{array}{l}\text { Seeds } \\
\text { per pod }\end{array}$ & $\begin{array}{c}\text { Seed } \\
\text { weight/ } \\
\text { pod }(\mathrm{g})\end{array}$ & \begin{tabular}{|c|}
$100-$ \\
seed \\
weight $(\mathrm{g})$
\end{tabular} & $\begin{array}{c}\text { Harvest } \\
\text { Index (\%) }\end{array}$ & $\begin{array}{r}\text { Seed yield } \\
\text { / plant }(\mathrm{g})\end{array}$ \\
\hline$\sigma^{2}$ gca & 0.76 & 0.72 & 0.01 & 0.12 & 0.04 & 0.04 & 3.51 & 0.29 & 0.01 & 0.00 & 0.00 & -0.05 & 0.07 \\
\hline$\sigma^{2} s c a$ & 5.05 & 4.27 & 0.08 & 7.01 & 0.19 & 0.15 & 4.79 & 4.36 & 0.11 & 0.00 & 0.12 & 16.53 & 1.32 \\
\hline$\sigma^{2} \mathrm{gca} / \sigma^{2} \mathrm{sca}$ & 0.15 & 0.17 & 0.06 & 0.01 & 0.23 & 0.26 & 0.73 & 0.07 & 0.01 & 0.00 & 0.02 & -0.00 & 0.05 \\
\hline$\sigma^{2} A$ & 1.53 & 1.43 & 0.01 & 0.24 & 0.09 & 0.08 & 7.03 & 0.57 & 0.01 & 0.00 & 0.01 & -0.69 & 0.13 \\
\hline$\sigma^{2} D$ & 5.05 & 4.27 & 0.08 & 7.01 & 0.19 & 0.15 & 4.79 & 4.36 & 0.11 & 0.00 & 0.12 & 16.53 & 1.32 \\
\hline$\sigma^{2} A / \sigma^{2} D$ & 0.302 & 0.34 & 0.13 & 0.03 & 0.46 & 0.53 & 1.46 & 0.13 & 0.12 & 1.264 & 0.06 & -0.01 & 0.10 \\
\hline $\begin{array}{c}\text { Degree of dom }= \\
\left(\sigma^{2} D / \sigma^{2} A\right)^{1 / 2}\end{array}$ & 1.82 & 1.73 & 2.74 & 5.41 & 1.48 & 1.37 & 0.83 & 2.76 & 2.83 & 0.89 & 3.98 & 13.31 & 3.14 \\
\hline
\end{tabular}


Table 3: Estimates of General combining ability (GCA) effects of parents for yield and its attributing characters in green gram

\begin{tabular}{|c|c|c|c|c|c|c|c|c|c|c|c|c|c|}
\hline Parents & $\begin{array}{c}\text { Days to } \\
50 \% \\
\text { flowering }\end{array}$ & $\begin{array}{l}\text { Days to } \\
\text { maturity }\end{array}$ & $\begin{array}{c}\text { Primary } \\
\text { branches/ } \\
\text { plant }\end{array}$ & $\begin{array}{c}\text { Plant } \\
\text { height } \\
(\mathrm{cm})\end{array}$ & $\begin{array}{l}\text { Pods/ } \\
\text { cluster }\end{array}$ & $\begin{array}{c}\text { Clusters } \\
\text { per plant }\end{array}$ & $\begin{array}{c}\text { Pods per } \\
\text { plant }\end{array}$ & $\begin{array}{c}\text { Biological } \\
\text { yield per } \\
\text { plant }\end{array}$ & $\begin{array}{c}\text { Seeds pe } \\
\text { pod }\end{array}$ & $\begin{array}{c}\text { Seed } \\
\text { weight/ } \\
\text { pod }(\mathrm{g})\end{array}$ & $\begin{array}{c}100-\text { seed } \\
\text { weight }(\mathrm{g})\end{array}$ & $\begin{array}{c}\text { Harvest } \\
\text { Index (\%) }\end{array}$ & $\begin{array}{c}\text { Seed } \\
\text { yield/plant } \\
\text { (g) }\end{array}$ \\
\hline \multicolumn{14}{|l|}{ Lines (Female) } \\
\hline JM 721 & -0.40 * & 1.50 ** & $0.06 \mathrm{~ns}$ & $-1.30 * *$ & $0.60 * *$ & $0.08 \mathrm{~ns}$ & 3.39 ** & $2.65^{* *}$ & 0.44 ** & $0.05^{* *}$ & 0.06 * & -0.62 ns & $0.97^{* *}$ \\
\hline BM 4 & $-2.65 * *$ & -1.33 ** & 0.18 ** & $-0.91 * *$ & $0.95^{* *}$ & 0.68 ** & 7.14 ** & 2.34 ** & $0.18 \mathrm{~ns}$ & 0.06 ** & $0.05 \mathrm{~ns}$ & 2.28 ** & $1.41^{* *}$ \\
\hline PKVAKM 4 & 0.51 ** & 2.42 ** & $0.12^{* *}$ & $1.61^{* *}$ & $0.39 * *$ & $1.13^{* *}$ & $6.89 * *$ & $0.48^{* *}$ & $0.00 \mathrm{~ns}$ & -0.02 ** & $0.17^{* *}$ & -1.54 ** & $0.02 \mathrm{~ns}$ \\
\hline TARM 2 & $1.18^{* *}$ & $-0.25 \mathrm{~ns}$ & $-0.44 * *$ & $-1.53 * *$ & $-0.79 * *$ & $-0.51^{* *}$ & -7.36 ** & $-2.30 * *$ & -0.32 ** & -0.04 ** & $-0.60 * *$ & $-2.09 * *$ & $-1.32 * *$ \\
\hline ML 131 & -0.99 ** & $-2.75^{* *}$ & $0.21^{* *}$ & $0.93^{* *}$ & -0.52 ** & $-0.57^{* *}$ & $-5.03^{* *}$ & -2.62 ** & -0.25 * & $-0.03^{* *}$ & $0.07^{* *}$ & $1.28^{* *}$ & $-0.99 * *$ \\
\hline JM 10 & $2.35^{* *}$ & 0.42 ** & $-0.13 * *$ & 1.19 ** & $-0.63 * *$ & $-0.82 * *$ & $-5.03 * *$ & $-0.55 * *$ & $-0.05 n s$ & -0.01 * & $0.25^{* *}$ & $0.68 \mathrm{~ns}$ & $-0.09 \mathrm{~ns}$ \\
\hline $\mathrm{SE}(\mathrm{gi}) \pm$ & 0.155 & 0.150 & 0.034 & 0.237 & 0.071 & 0.081 & 0.590 & 0.087 & 0.095 & 0.002 & 0.025 & 0.388 & 0.049 \\
\hline SE (gi-gj) \pm & 0.219 & 0.212 & 0.048 & 0.335 & 0.100 & 0.115 & 0.836 & 0.124 & 0.134 & 0.004 & 0.035 & 0.549 & 0.069 \\
\hline \multicolumn{14}{|l|}{ Testers (Male) } \\
\hline AKM 8802 & $3.43^{* *}$ & $2.17^{* *}$ & $-0.27^{* *}$ & $1.25^{* *}$ & $0.54^{* *}$ & $0.33^{* *}$ & 5.03 ** & $1.41^{* *}$ & 0.36 ** & 0.01 ** & -0.04 * & $2.13^{* *}$ & 1.00 ** \\
\hline TJM3 & -4.74 ** & $-4.72 * *$ & 0.22 ** & $-1.18^{* *}$ & $0.17^{* *}$ & -0.32 ** & $-0.47 \mathrm{~ns}$ & $-0.15^{*}$ & $-0.17^{*}$ & $0.00 \mathrm{~ns}$ & $0.08^{* *}$ & -1.08 ** & $-0.25^{* *}$ \\
\hline PDM 1 & 0.26 * & $1.11^{* *}$ & -0.12 ** & $1.73^{* *}$ & $-0.53^{* *}$ & $-0.23^{* *}$ & -4.19 ** & $0.02 \mathrm{~ns}$ & $-0.31^{* *}$ & $-0.01 * *$ & $-0.17^{* *}$ & $-2.05^{* *}$ & $-0.31^{* *}$ \\
\hline HUM 1 & 1.04 ** & $1.44^{* *}$ & $0.17^{* *}$ & $-1.81^{* *}$ & $-0.18^{* *}$ & 0.22 ** & $-0.36 n s$ & $-1.27^{* *}$ & $0.12 \mathrm{~ns}$ & -0.00 * & $0.13^{* *}$ & 1.00 ** & $-0.44 * *$ \\
\hline SE (gi) \pm & 0.126 & 0.122 & 0.027 & 0.193 & 0.058 & 0.066 & 0.483 & 0.071 & 0.077 & 0.002 & 0.020 & 0.317 & 0.040 \\
\hline SE (gi-gj) \pm & 0.179 & 0.173 & 0.039 & 0.273 & 0.082 & 0.094 & 0.683 & 0.101 & 0.109 & 0.003 & 0.029 & 0.448 & 0.056 \\
\hline
\end{tabular}


Table 4: Estimates of Specific combining ability (SCA) effects of 24 hybrids for yield and its attributing characters in green gram

\begin{tabular}{|c|c|c|c|c|c|c|c|c|c|c|c|c|c|}
\hline Parents & $\begin{array}{c}\text { Days to } \\
50 \% \\
\text { flowering } \\
\end{array}$ & $\begin{array}{l}\text { Days to } \\
\text { maturity }\end{array}$ & $\begin{array}{c}\text { Primary } \\
\text { branches } \\
\text { /plant }\end{array}$ & $\begin{array}{c}\text { Plant } \\
\text { height } \\
(\mathrm{cm})\end{array}$ & $\begin{array}{c}\text { Pods/ } \\
\text { cluster }\end{array}$ & $\begin{array}{c}\text { Clusters } \\
\text { per plant }\end{array}$ & $\begin{array}{l}\text { Pods } \\
\text { Per } \\
\text { plant }\end{array}$ & $\begin{array}{c}\text { Biological } \\
\text { yield per } \\
\text { plant }\end{array}$ & $\begin{array}{c}\text { Seeds } \\
\text { per } \\
\text { pod }\end{array}$ & $\begin{array}{c}\text { Seed } \\
\text { weight/ } \\
\operatorname{pod}(\mathrm{g})\end{array}$ & \begin{tabular}{|c|}
$\begin{array}{c}100 \text {-seed } \\
\text { weight } \\
(\mathrm{g})\end{array}$ \\
\end{tabular} & $\begin{array}{c}\text { Harvest } \\
\text { Index } \\
(\%) \\
\end{array}$ & $\begin{array}{l}\text { Seed } \\
\text { yield/ } \\
\text { plant }(\mathrm{g})\end{array}$ \\
\hline JM $721 \times$ AKM 8802 & $3.57^{* *}$ & $0.50 \mathrm{~ns}$ & $-0.52^{* *}$ & $0.60 \mathrm{~ns}$ & $0.59^{* *}$ & $0.10 \mathrm{~ns}$ & 2.39 * & $2.78^{* *}$ & $0.00 \mathrm{~ns}$ & $0.02^{* *}$ & $0.16^{* *}$ & $-3.09^{* *}$ & $0.63^{\star \star}$ \\
\hline JM721 x TJM 3 & $-2.93^{* *}$ & -2.28 ** & $0.02 n s$ & $0.06 \mathrm{~ns}$ & $0.20 \mathrm{~ns}$ & $-0.22 n s$ & $-0.78 \mathrm{~ns}$ & -1.96 ** & $0.21 \mathrm{~ns}$ & $-0.01 \mathrm{~ns}$ & $0.04 \mathrm{~ns}$ & $0.03 \mathrm{~ns}$ & $-0.78^{* *}$ \\
\hline JM721 x PDM 1 & $0.74^{*}$ & $2.56^{* *}$ & $-0.04 n s$ & $2.45^{* *}$ & $0.09 \mathrm{~ns}$ & $0.03 \mathrm{~ns}$ & $1.61 \mathrm{~ns}$ & $1.87^{* *}$ & $-0.49 *$ & $0.00 \mathrm{~ns}$ & $0.05 \mathrm{~ns}$ & $0.01 \mathrm{~ns}$ & $0.74^{* *}$ \\
\hline JM721 x HUM 1 & -1.38 ** & -0.78 * & $0.54^{\star \star}$ & $-3.11^{\star *}$ & $-0.88^{* *}$ & $0.08 \mathrm{~ns}$ & $-3.22^{* *}$ & -2.68 ** & $0.28 \mathrm{~ns}$ & $-0.01 \mathrm{~ns}$ & $-0.25^{\star *}$ & $3.05^{* *}$ & $-0.59 * *$ \\
\hline BM 4 x AKM 8802 & $2.49 * *$ & $1.00^{* *}$ & $-0.20^{\star *}$ & $-6.06^{\star \star}$ & $-0.03 n s$ & $-0.32 n s$ & $0.64 \mathrm{~ns}$ & $-3.08^{* *}$ & $-0.37 n s$ & $0.02 * *$ & $0.00 \mathrm{~ns}$ & $-1.51 \mathrm{~ns}$ & -1.64 ** \\
\hline BM 4 x TJM 3 & 1.32 ** & $0.22 \mathrm{~ns}$ & $0.37^{\star \star}$ & $2.57^{\star *}$ & $0.21 \mathrm{~ns}$ & $-0.38 *$ & $-0.53 \mathrm{~ns}$ & $0.48^{* *}$ & $0.50 *$ & $0.00 \mathrm{~ns}$ & -0.19 ** & $-0.54 \mathrm{~ns}$ & $0.11 \mathrm{~ns}$ \\
\hline 3M 4 x PDM 1 & -2.01 ** & -1.28 ** & $-0.05 n s$ & $2.12^{* *}$ & 0.33 * & $-0.03 n s$ & $0.86 \mathrm{~ns}$ & 3.21 ** & $0.33 \mathrm{~ns}$ & $0.00 \mathrm{~ns}$ & 0.33 ** & $1.06 \mathrm{~ns}$ & $1.60^{* *}$ \\
\hline BM 4 x HUM 1 & -1.79 ** & $0.06 \mathrm{~ns}$ & $-0.11 n s$ & $1.37^{* *}$ & $-0.51^{* *}$ & $0.72^{* *}$ & $-0.97 \mathrm{~ns}$ & $-0.60^{* *}$ & $-0.46^{*}$ & -0.02 ** & $-0.14^{* *}$ & $0.99 \mathrm{~ns}$ & $-0.07 n s$ \\
\hline PKVAKM4xAKM8802 & $-1.01^{* *}$ & $1.92 * *$ & 0.39 ** & $1.46^{* *}$ & $0.20 \mathrm{~ns}$ & $0.32 \mathrm{~ns}$ & 2.89 * & $1.78^{* *}$ & $0.44^{*}$ & $-0.01 \mathrm{~ns}$ & $-0.02 n s$ & $4.77^{* *}$ & $1.65^{* *}$ \\
\hline PKVAKM 4 x TJM 3 & $-0.85^{* *}$ & -2.19 ** & $-0.14^{*}$ & $-2.22^{* *}$ & $-0.16 n s$ & 10 * & $-0.28 \mathrm{~ns}$ & $0.17 \mathrm{~ns}$ & $-0.26 n s$ & $-0.01 \mathrm{~ns}$ & $-0.14^{* *}$ & $1.04 \mathrm{~ns}$ & $0.17 \mathrm{~ns}$ \\
\hline PKVAKM 4 x PDM 1 & $-0.18 n s$ & $-0.69 *$ & $-0.06 n s$ & $-0.36 n s$ & $-0.67^{* *}$ & $-0.55^{\star *}$ & $-4.89^{\star *}$ & -2.90 ** & $-0.13 n s$ & $0.01 \mathrm{~ns}$ & $0.08 \mathrm{~ns}$ & $-1.84 *$ & -1.54 ** \\
\hline PKVAKM 4 x HUM 1 & $2.04^{* *}$ & $0.97^{* *}$ & $-0.19^{* *}$ & $1.12 *$ & 0.62 ** & $-0.17 n s$ & $2.28 \mathrm{~ns}$ & $0.95^{* *}$ & $-0.05 n s$ & $0.01 \mathrm{~ns}$ & $0.08 \mathrm{~ns}$ & $-3.97^{* *}$ & -0.28 ** \\
\hline TARM 2 x AKM 8802 & $-1.68^{* *}$ & $0.58 \mathrm{~ns}$ & $-0.11 n s$ & $2.77^{\star *}$ & $-0.19 n s$ & $-0.30 \mathrm{~ns}$ & $-1.19 \mathrm{~ns}$ & $0.05 \mathrm{~ns}$ & $-0.41 *$ & $-0.02^{* *}$ & $0.21^{\star \star}$ & -2.92 ** & $-0.51^{\star *}$ \\
\hline TARM 2 x TJM 3 & 3.49 ** & $3.47^{* *}$ & $-0.01 n s$ & $-2.64^{* *}$ & $-0.12 n s$ & $-0.46^{* *}$ & $-2.03 n s$ & $0.75^{* *}$ & $-0.13 n s$ & $0.00 \mathrm{~ns}$ & $0.07 \mathrm{~ns}$ & 2.50 ** & $0.71^{* *}$ \\
\hline TARM 2 x PDM 1 & $-0.51 \mathrm{~ns}$ & -1.36 ** & $0.04 \mathrm{~ns}$ & $-1.98^{\star *}$ & $0.01 \mathrm{~ns}$ & $0.66^{* *}$ & $1.03 \mathrm{~ns}$ & $-0.39 *$ & $0.37 \mathrm{~ns}$ & $-0.00 \mathrm{~ns}$ & $0.01 \mathrm{~ns}$ & $-0.05 n s$ & $-0.13 n s$ \\
\hline TARM 2 x HUM 1 & -1.29 ** & -2.69 ** & $0.08 n s$ & $1.86^{* *}$ & 0.30 * & $0.11 \mathrm{~ns}$ & $2.19 \mathrm{~ns}$ & $-0.40 *$ & $0.17 \mathrm{~ns}$ & $0.02^{* *}$ & -0.29 ** & $0.46 \mathrm{~ns}$ & $-0.07 n s$ \\
\hline ML131 x AKM 8802 & $-2.51^{\star *}$ & -2.92 ** & $0.34^{\star *}$ & $1.60^{\star *}$ & $-0.32 *$ & $0.12 \mathrm{~ns}$ & $-2.53 *$ & -1.32 ** & $0.15 \mathrm{~ns}$ & $-0.02^{* *}$ & -0.79 ** & $-6.30^{* *}$ & $-1.53^{* \star}$ \\
\hline ML131 x TJM 3 & $0.65 *$ & $1.97^{* *}$ & $-0.12 n s$ & 1.39 ** & $0.12 \mathrm{~ns}$ & $0.33^{*}$ & 2.64 * & 1.00 ** & $0.16 \mathrm{~ns}$ & $0.01 \mathrm{~ns}$ & $0.39^{* *}$ & $-0.71 \mathrm{~ns}$ & 0.38 ** \\
\hline ML131 x PDM 1 & $0.65^{*}$ & $0.14 \mathrm{~ns}$ & $0.09 \mathrm{~ns}$ & $-0.98 *$ & $0.18 \mathrm{~ns}$ & $-0.08 n s$ & $1.03 \mathrm{~ns}$ & $-0.33 \mathrm{~ns}$ & $-0.07 n s$ & $0.01 \mathrm{~ns}$ & $0.07 \mathrm{~ns}$ & 3.49 ** & $0.38^{* *}$ \\
\hline ML131 x HUM 1 & 1.21 ** & $0.81 * *$ & $-0.31^{* *}$ & $-2.01^{* *}$ & $0.03 \mathrm{~ns}$ & $-0.37^{*}$ & $-1.14 \mathrm{~ns}$ & $0.65^{* *}$ & $-0.24 n s$ & $0.01 \mathrm{~ns}$ & 0.34 ** & $3.52^{* *}$ & $0.77^{* *}$ \\
\hline JM 10 x AKM 8802 & $-0.85^{* *}$ & -1.08 ** & $0.11 \mathrm{~ns}$ & $-0.36 n s$ & $-0.25 n s$ & 0.08 ns & $-2.19 n s$ & $-0.20 \mathrm{~ns}$ & $0.19 \mathrm{~ns}$ & 0.02 ** & 0.44 ** & $9.04^{* *}$ & $1.40^{* *}$ \\
\hline JM 10 x TJM 3 & -1.68 ** & -1.19 ** & $-0.12 n s$ & $0.83 \mathrm{~ns}$ & $-0.24 n s$ & $0.32 \mathrm{~ns}$ & $0.97 \mathrm{~ns}$ & $-0.44^{*}$ & $-0.48^{*}$ & $-0.00 \mathrm{~ns}$ & -0.16 ** & -2.32 ** & -0.58 ** \\
\hline JM 10 x PDM 1 & 1.32 ** & 0.64 * & $0.02 \mathrm{~ns}$ & $-1.24 *$ & $0.05 \mathrm{~ns}$ & $-0.03 n s$ & $0.36 \mathrm{~ns}$ & $-1.44^{* *}$ & $-0.01 \mathrm{~ns}$ & $-0.01 \mathrm{~ns}$ & -0.54 ** & $-2.67^{* *}$ & $-1.06^{* *}$ \\
\hline JM 10 x HUM 1 & $1.21 * *$ & 1.64 ** & $-0.01 n s$ & $0.77 \mathrm{~ns}$ & 0.44 ** & -0.38 * & $0.86 \mathrm{~ns}$ & 2.08 ** & $0.30 \mathrm{~ns}$ & $-0.00 \mathrm{~ns}$ & 0.26 ** & $-4.05^{* *}$ & $0.24 *$ \\
\hline SE (Sij) & 0.310 & 0.300 & 0.068 & 0.474 & 0.142 & 0.163 & 1.183 & 0.175 & 0.190 & 0.005 & 0.050 & 0.776 & 0.097 \\
\hline SE (Sij-Skl) & 0.439 & 0.424 & 0.096 & 0.670 & 0.201 & 0.230 & 1.673 & 0.248 & 0.269 & 0.008 & 0.071 & 1.098 & 0.138 \\
\hline
\end{tabular}

${ }^{*}$ Significant at $5 \%$ level, ${ }^{*}$ Significant at $1 \%$ level 
The estimates of gca effects (Table 3) further indicated that the parental lines showing high gca effects for seed yield also exhibited high to average gca effects for most of the yield components. Similar results were reported by Sunil et al. (2010), Sathya and Jayamani (2011), Sujatha and Kajjidoni (2013) and Suresh (2014). The best combiner line was BM4 followed by JM 721. These parents can be intensively used in the hybridization programme aimed at the amelioration of seed yield. Among the testers, AKM8802 was the best general combiner for most of the traits, viz., plant height, number of branches/ plant, clusters/plant, pods/cluster, pods/plant, seeds/pods, harvest index and seed yield/plant. A combination of per se performance and gca effects would result in the selection of potential parents with a good reservoir of superior genes.

Specific combining ability estimates revealed a very wide range of variation for all the characters (Table 4). High sca effects are mostly revealed due to the dominance and interaction effects existed between the hybridizing parents. In green gram, owing to its autogamous genetic nature, sca effects are not readily useful. However, if sca is due to additive $\times$ additive gene action, it is fixable at later generations and superior transgressive segregants can be isolated from such crosses. In the present study, the cross PKVAKM4 x AKM8802 recorded the highest and significant sca effect for seed yield per plant and significantly high for harvest index, pods per plant, plant height and the number of branches/plant. Among other crosses with high and significant sca effects for seed yield per plant are BM4 x PDM1, JM 10 x AKM 8802, JM $721 \times$ PDM1, ML131 x HUM 1 and TARM $2 \times$ TJM 3. The crosses desirable for earliness with significant sca effect are JM721 x TJM 3, BM4 x PDM1, ML131 x AKM8802, TARM2 x HUM1 and JM10 x TJM3. These crosses could be exploited effectively through heterosis breeding programme for getting desirable recombinants from the segregating population.

The genetic analysis revealed that the seed yield/plant and most of its important component characters have shown preponderance of non-additive genetic variance having considerable amount of additive gene action in their expression. In self pollinated crops, it has been generally observed that additive genetic variance gets fixed rapidly. Therefore, seed yield / plant and its component traits in green gram can be modified desirably by recurrent selection in progenies derived by intermating selected plants from segregating generations. This would ultimately result in aggregation of favorable genes and break undesirable linkage. Hence, improvement in yield might be expected in later generations as the dominance and epistatic gene interactions would disappear and additive fixable genes will be restored by inter-mating of segregating population and recurrent selection. Crosses namely PKVAKM4 x AKM8802, BM4 x PDM1, JM 10 x AKM 8802, JM 721 x PDM1, ML131 x HUM 1 and TARM $2 \times$ TJM 3 having high SCA may be used for growing advanced generations for desirable yield improvement and JM721 $\mathrm{x}$ TJM 3, BM4 x PDM1, ML131 x AKM8802, TARM2 $\times$ HUM1 and JM10 x TJM3 desirable for earliness. The plant improvement methods viz., bi-parental mating, line $x$ tester or diallel selective mating, thereafter the selection following pedigree method may give a better achievement towards yield improvement.

\section{REFERENCES}

Anbumalarmathi, J., Rangasamy, P. and Yogameenakshi, P. (2005) Studies on combining ability analysis for quality traits in mungbean (Vigna radiata (L.)Wilczek).Madras Agriculture Journal 92(4-6): 188-192.

Bainade, P. S., Manjare, M. R., Deshmukh, S. G. and Kumbhar S. D. (2014) Genetic analysis in green gram [Vigna radiata (L.) Wilczek] subjected to North Carolina

mating design-1. The BioScan 9(2): 875878.

Barad, H. R., Pithia, M. S. and Vachhani, J. H. (2008) Heterosis and combining ability studies for economic traits in genetically diverse lines of mungbean (Vigna radiata (L.) Wilczek).Legume Research 31(1): 68-71.

Bhavani, R.T., Chauhan, R.M.., Patel, J.R., Patel, D.K., \& Patel, R.A. (2016) 
Combining ability studies for grain yield and architectural traits in mungbean [architectural traits in mungbean [Vigna radiata L.) Wilczek].The BioScan 11(3):1871-1876.

Kempthorne, O. (1957)An introduction to genetic statistics. John Wiley and Sons, New York.

Marappa, N. (2008) Line x Tester analysis in mungbean [Vigna radiata (L.) Wilczek].Asian Journal of Bio Sciences 3(2): 289-294.

Narsimhulu, R., Naidu, N. V., Reddy, K. H., \& Naidu, G. M. (2014) Combining ability for yield attributes in green gram (Vigna radiata L. Wilczek). The BioScan 9 (4), 1167-1671.

Sathya, M. and Jayamani, P. (2011) Heterosis and combining ability studies in green gram.Journal of Food Legumes 24(4): 282-287.
Sujatha, K. and Kajjidoni, S. T. (2013) Genetic analysis involving selected powdery mildew resistant lines in mungbean (Vigna radiata (L.) Wilczek).Molecular Plant Breeding 4(5): 38-43.

Sunil, K. B., Prakash, M. and Gokul Krishna, J. (2010) Combining ability analysis in mungbean (Vigna radiata (L.) Wilczek).Crop Improvement 37(2): 165167.

Suresh (2014) Combining ability analysis for yield and yield component traits in mungbean [Vigna radiata (L.) Wilczek].2nd International Conference on Agricultural and Horticultural Sciences Feb.pp. 0305.

Swarna Latha, V., Eswari, K.B. and Sudheer Kumar, S. (2018) Combining ability analysis for seed yield and its component characters in greengram (Vigna radiata (L.) Wilczek.).International Journal of Chemical Studies 6(2): 237-242. 